This item was submitted to Loughborough's Research Repository by the author.

Items in Figshare are protected by copyright, with all rights reserved, unless otherwise indicated.

\title{
Robotic-assisted internal fixation of femoral fractures
}

PLEASE CITE THE PUBLISHED VERSION

http://dx.doi.org/10.1243/PIME_PROC_1995_209_316_02

\section{PUBLISHER}

Professional Engineering Publishing / @ IMechE

VERSION

VoR (Version of Record)

LICENCE

CC BY-NC-ND 4.0

REPOSITORY RECORD

Bouazza-Marouf, Kaddour, lan Browbank, and James R. Hewit. 2019. "Robotic-assisted Internal Fixation of Femoral Fractures". figshare. https://hdl.handle.net/2134/14127. 


\title{
Robotic-assisted internal fixation of femoral fractures
}

\author{
K Bouazza-Marouf, BSc, PhD, CEng, MIMechE, I Browbank, BEng, MSc and J R Hewit,* BSc, MSc, PhD, CEng, MIEE, \\ FIMechE, FRSA
}

Department of Mechanical Engineering, Loughborough University of Technology

\begin{abstract}
Closed surgical techniques for the internal fixation of femoral fractures require orthopaedic surgeons to work in close proximity to $X$-rays. In addition to the occupational health risk this imposes, inexperienced surgeons often encounter great difficulty in achieving optimal positioning of fracture repair fixtures. A vision-guided robotic system has been proposed as a possible solution to these problems and an initial investigation involving two exemplar orthopaedic procedures has been undertaken Robotic surgery assistance imposes rigorous safety-related design constraints, since the orthopaedic robot must operate in close proximity to the patient and operating staff. The design and implementation of a purpose-built robotic system for orthopaedic surgery assistance is described in this paper.
\end{abstract}

Key words: robotic-assisted surgery, orthopaedics, femoral fractures

\section{INTRODUCTION}

The use of X-ray imaging for diagnosis and clinical evaluation is an essential part of the practice of orthopaedic surgery. In addition to the direct exposure received by the patient, the surgeon and theatre staff are also exposed to a low dose of $\mathrm{X}$-ray radiation during surgical procedures. In the past, the health risks associated with these $\mathrm{X}$-ray exposures were deemed to be minimal given that the patient is unlikely to undergo regular examination and that the surgical team observe relevant safety procedures such as the wearing of protective lead aprons.

In the past few years, however, changes in clinical practice towards the use of 'closed' internal fixation of fractures have given cause for concern. These techniques, which de not require the fracture site to be opened up, have found widespread use due to the benefits of lower intra-operative blood loss, reduced infections at the fracture site and reduced risk of wound complications which results from the use of smaller incisions. However, as there is no direct visualization of the fracture site, an extensive use of X-ray fluoroscopy is necessary to monitor the progress of these procedures. As such, the surgeon's hands and body are unavoidably brought into close proximity to the $\mathrm{X}$-ray beam, significantly increasing the radiation dose he or she receives.

In view of the concern this occupational health hazard has raised, several quantitative studies have been carried out to measure the levels of exposure experienced by orthopaedic surgeons over a one year period, or more specifically during particular surgical techniques $(1,2)$. All such studies have found that the radiation dose the surgeons receive is within the currently recommended guidelines. However, the accumulative effects of long-term low-dose exposure are not well understood. A number of independent authors have, therefore, concluded that it is wise to work on the basis that there is no safe dose of radiation.

Exposure to radiation obeys an inverse square law and as such decreases as the distance from the source increases. Therefore, a satisfactory solution to the X-ray safety problem requires the surgeon to be remote from

This paper was presented at the IMechE seminar on 'Robotics in keyhole surgery' on 5 May 1993. The MS was received on 23 June 1994 and was accepted for publication on 16 December 1994.

* Now at Department of APEME, University of Dundee. the patient at the time of imaging. This safe, 'remote', surgery ability could be achieved through the use of a robotic manipulator under the surgeon's control (3). In addition to X-ray safety, the robotic solution offers the advantages of increased surgical precision without major changes to current surgical practices being necessary.

The main objectives of the proposed robot-assisted surgery are, therefore, the removal of the surgeon from the X-ray radiation hazard and the improvement of the success rate of orthopaedic surgical procedures.

Comprehensive reviews of robotic applications in medicine and healthcare may be found in Preising $e t$ al. (4) and Kassler (5). In the majority of these applications commercially-available industrial robots have been used, with additional design features incorporated to satisfy the safety and hygiene specifications necessary for such applications. Extensive modification of commercial industrial robots is not the optimal approach to robotic-assisted surgery. In addition to the high cost of such modification, it is debatable whether industrial robots could be made fully safe for assistance in invasive surgery.

The aim of this project is the design of a novel robotic device, under the remote control of the surgeon, to perform invasive surgery. This robotic device is to be used for those parts of orthopaedic operations where location and drilling are performed using $\mathbf{X}$-ray image feedback. Two procedures (described in Section 2) for the internal fixation of fractured femurs have been selected here as exemplar orthopaedic repair procedures. It is hoped that this research will ultimately demonstrate the true potential of the robotic surgical assistant, reducing operating times, speeding up post-operative recovery, and improving procedure accuracy and hence success rate.

In today's financial climate, and unlike such applications as neurosurgery, cost will be a limiting factor for the acceptance of this robotic system by the medical community. Therefore, the aim of this project is to develop a cost-effective design taking into account safety and hygiene constraints, to make use of existing $\mathrm{C}$-arm X-ray facilities for the acquisition of images, and for the robotic system to be fully compatible with the operating theatre environment without the need for 
modifications. This will minimize the total cost of implementation of this robotic system.

\section{EXEMPLAR ORTHOPAEDIC REPAIR PROCEDURES}

Femoral fractures are generally categorized as being either proximal or shaft fractures. The term 'proximal' refers to a fracture at the upper end of the femur, and encompasses both femur neck and trochanteric fractures. Femoral neck fractures are almost entirely confined to elderly patients (6), whose bones are weakened by osteoporosis, and often occur as a result of a fall. Unfortunately, as the average age of the population increases, the incidence of these fractures is also expected to rise sharply. Trochanteric and shaft fractures can occur during road traffic accidents or as sports-related injuries and result from twisting or a direct blow to the femur.

To facilitate early mobilization of the patient, thus avoiding secondary complications related to prolonged bed rest, current orthopaedic fracture repair procedures involve the use of internal fixation. The metal implants inserted during these surgeries stabilize the fracture site thus allowing the natural bone healing process to occur. Following radiographic verification that the fracture has healed, the implant may then be removed at a later date.

\subsection{Repair of proximal femoral fractures}

The repair of trochanteric and, to a lesser extent, femur neck fractures involves the use of a sliding screw and plate as shown in Fig. 1. The main difficulty associated with the insertion of this fixture lies in ensuring that the screwed rod is located at the correct orientation and inserted to the correct extent. If either of these is incorrect, failure of the fixture may occur due to the screw

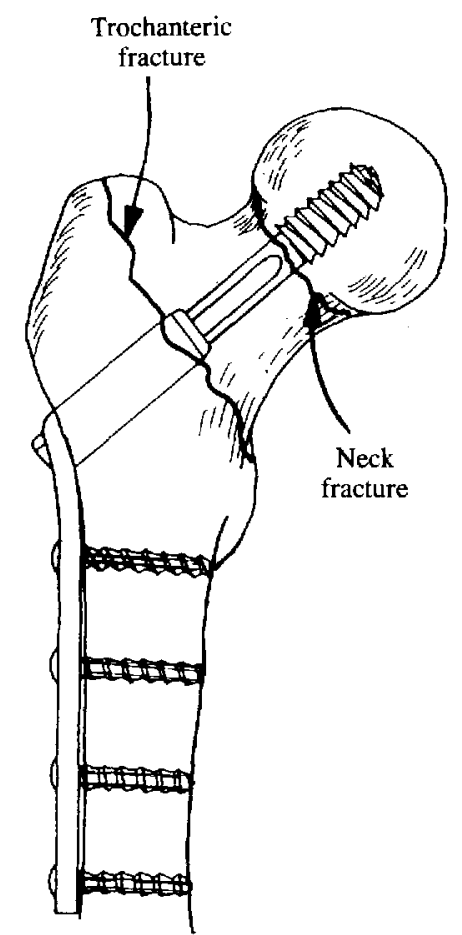

Fig. I Fixation of proximal femoral fractures using screw and plate (both trochanteric and neck fractures are shown) cutting-out of the femoral head, when the load of walking is applied. The success of the repair depends, therefore, on the correct location of the screwed rod.

In order to ensure correct location of the sliding hip screw component, the surgeon initially inserts a pilot drill bit (or guide-wire) up the neck of the femur usually at an angle of $135^{\circ}$ to the femoral shaft. The guide-wire is then left in position and used as a guide during the drilling and insertion of the screwed rod. The complete process is currently accomplished manually by the orthopaedic surgeon, using X-rays to obtain location and orientation information. The surgeon encounters difficulties in the insertion of the guide-wire. Given the 'blind' nature of the insertion, the surgeon is forced to take many $\mathrm{X}$-ray images to monitor progress during the drilling of the pilot hole, and multiple attempts may often be required before a satisfactory location of the guide-wire is obtained. This results in a weakening of the bone, often resulting in a less than optimum location having to be accepted, and increased radiation exposure of the operating staff and patient due to the extended operating time.

The surgical techniques currently used to repair proximal femoral fractures involve extensive use of C-frame fluoroscopy to check fixture location inside the femur. Although it is standard practice for the surgeon to wear a heavy lead apron, which is restrictive and tiring, the hands are often left unprotected while holding the drill during the location/insertion of the guide-wire. The surgeon is therefore unavoidably exposed to a low dose of X-ray radiation.

\subsection{Repair of femoral shaft fractures}

The long intramedullary locking nail is quickly becoming the standard internal fixation treatment for femoral shaft fractures. Intramedullary nails are hollow stainless steel tubes which are inserted down the hand-reamed centre of the femur (medullary cavity) through a point of introduction at the proximal end of the bone. The incorporation into the nail's design of locking screws to control rotation, as shown in Fig. 2, allows excellent stabilization of the fracture site to be achieved (7).

The main problem associated with locked femoral nailing is the 'blind' insertion of the distal locking screws. These screws must pass through one bone cortex, the unseen holes in both sides of the distal nail and finally the second cortex in order to interlock the bone and the nail. Their location is significantly hampered by the fact that the open-section nail often distorts due to the torques and forces applied during manual insertion, thus preventing the reliable use of mechanical jigs. To overcome these difficulties, surgeons are currently using hand-held distal aiming devices in conjunction with $\mathrm{X}$-ray image intensification. Unfortunately, during the use of these targeting systems, the surgeon's hands are inevitably exposed to X-ray radiation.

\section{ROBOTIC SYSTEM}

The robotic system with the necessary interfaces is shown in a diagrammatical form in Fig. 3 and in a block diagram form in Fig. 4. It consists of a manipulator, a robot controller and a standard C-arm X-ray unit. No modification to the operating theatre 


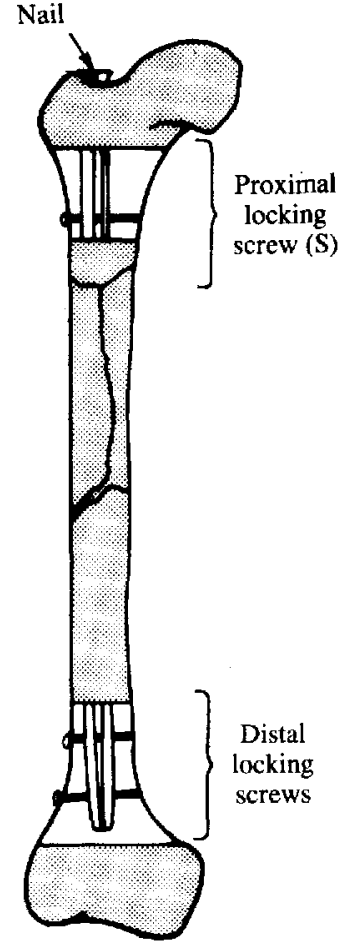

Fig. 2 Internal fixation of femoral shaft fracture using intramedullary nail

equipment is necessary for the implementation of this robotic system.

The manipulator used for the positioning and insertion of the drill bit is discussed in Subsection 3.1. The computer control system consists of PC-based robot controller and digital image processing system. The robot controller, comprising a personal computer with the necessary interface electronics and control-andsafety software, provides the servocontrol for the manipulator for the orientation and travel of the endeffector (or tool holder) and safety monitoring. The digital image processing system (DIPS) consists of a frame grabber, a display monitor and supporting imageprocessing software. The frame grabber is directly linked to the $\mathrm{X}$-ray unit via a video cable; this permits both the acquisition and storage of fluoroscopic images. The acquisition and processing of these images is discussed in Subsection 3.2.

The robotic-assisted orthopaedic surgery starts with positioning a calibration frame around the body part of interest. The calibration frame can be attached to the manipulator end-effector and is made up of two pairs of Perspex planes containing metallic balls and lead-wire crosses. Two near-orthogonal X-ray images are taken of the body part of interest together with the reference frame. These images are stored by the frame grabber and then displayed on the DIPS monitor. A straight line is drawn by the surgeon on each of the two images, indicating the path of the drill bit. This straight line path is then defined relative to a reference coordinate frame on the calibration frame. With the latter frame attached to the end-effector, the transformation matrix relating the reference coordinate frame to the manipulator is easily computed. Using this transformation matrix, the location of the straight line path with respect to the manipulator can be derived. The latter data are used to compute the manipulator joint commands.

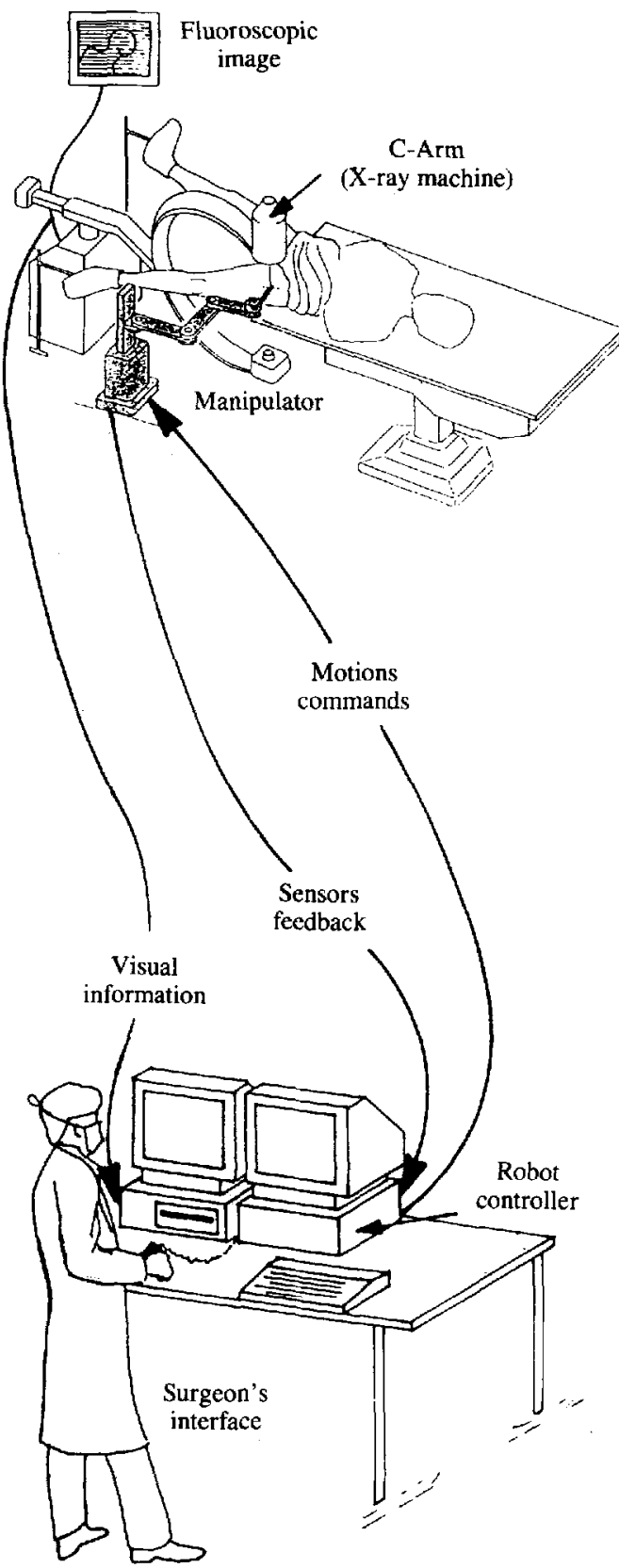

Fig. 3 Robotic-assisted orthopaedic surgery

As an example of robotic assistance in orthopaedic surgery, the robotic-assisted surgical procedure for the insertion of a guide-wire, used in the repair of proximal femur fractures, is detailed as follows:

1. The fracture is reduced by the surgeon, that is, the broken bone parts are restored to their natural position.

2. The manipulator is coarsely positioned near the fractured bone and the calibration frame is positioned around the hip.

3. Two near-orthogonal $\mathrm{X}$-ray images are taken using the $\mathrm{C}$-arm and stored by the frame grabber.

4. Internal and external calibrations are carried out (as defined in Subsection 3.2).

5. The calibration frame is removed.

6. The incision is made

7. The path of the guide-wire is indicated by the surgeon on each of the $\mathrm{X}$-ray images which are displayed, one at a time, on the DIPS. 


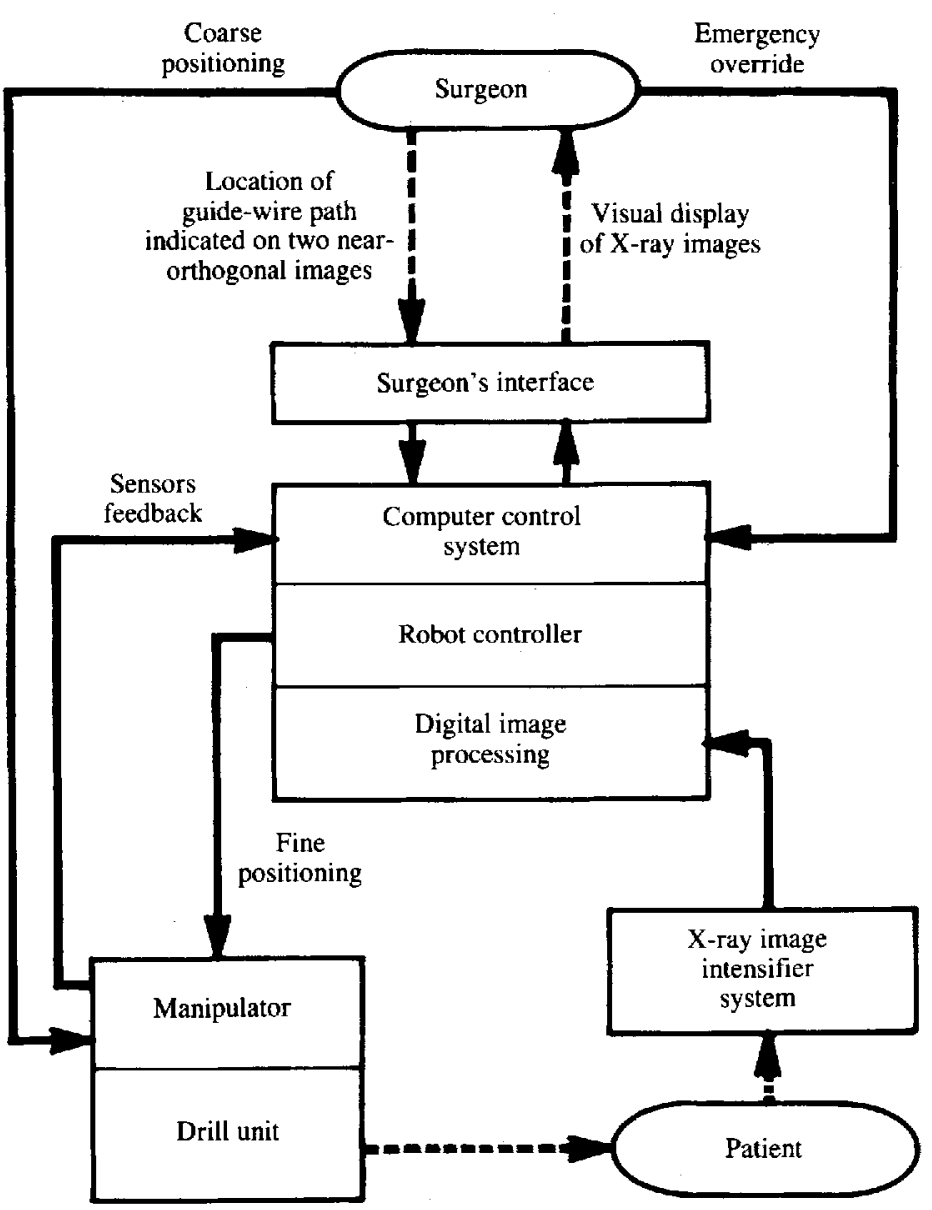

Fig. 4 Robotic system for the insertion of a guide-wire

8. The trajectory of the guide-wire is displayed for the surgeon to check and confirm.

9. The manipulator joint commands are computed and the drill-holder driven to a standby drilling position.

10. All the joints, except the drill-holder feed joint, are locked in position.

11. The drilling is initiated by the surgeon.

12. Another pair of $X$-ray images is taken for the surgeon to check/confirm the trajectory of the guide-wire.

It must be noted, however, that this project is not intended to design a robotic system which is solely dedicated to the two surgical procedures discussed in Section 2, as changes in clinical practice would soon render the device obsolete. The prime objective of this research is the provision of a generic image-guided surgical robot for assistance in orthopaedic repair procedures of fractured bones which are joined together using plates, screws or nails. The robotic system is aimed initially at, but not limited to, the two exemplar procedures. Other applications include surgical procedures which require increased precision, minimally invasive access, reduced trauma to the patient and/or elimination of radiation hazards to the surgeon. One example is the performing of needle biopsies.

\subsection{Prototype manipulator}

A five degrees-of-freedom (DOF) prototype manipulator has been designed and manufactured, integrated to a vision system and interfaced to a controlling com- puter. Factors taken into consideration while drawing up the specification for this manipulator included the safety of the patient and surgeon, precisional requirements, physical size, sterility and cost.

The manipulator's five DOF constitute horizontal (joint 1), vertical (joint 2), pan (joint 3), tilt (joint 4) and feed (joint 5) motions as shown in the schematic diagram in Fig. 5. Four DOF, joints 1 to 4 , are used for the positioning of the guide-wire along the required drill trajectory. The fifth DOF, joint 5 , is used during the insertion of the guide-wire into the femur.

Stepper motors have been used for the actuation of all the joints. The pan motion is driven through a worm-and-wheel gearbox fitted with a backlash compensation system; while the other four DOF are driven through backlash-free leadscrews.

The joints are instrumented using angular and linear potentiometers. Limit switches are fitted to all the joints to protect against mechanical overdrive. Software imposed limits have also been added, as a back up, to stop the motion before a limit switch is activated, and thus to avoid the resetting of the system which would be necessary once a limit switch is activated.

Given that the aim of this project is the development of an orthopaedic robot which can perform invasive surgery, the forces of interest are those resulting from the drilling into bones by the manipulator. Thus, the drill thrust force (or reaction force on the manipulator) must be taken into account at the design stage. This force depends on the mechanical properties of the bone, the drill bit used, the feed (or penetration) rate and the rotational speed of the drill bit. The forces obtained in 


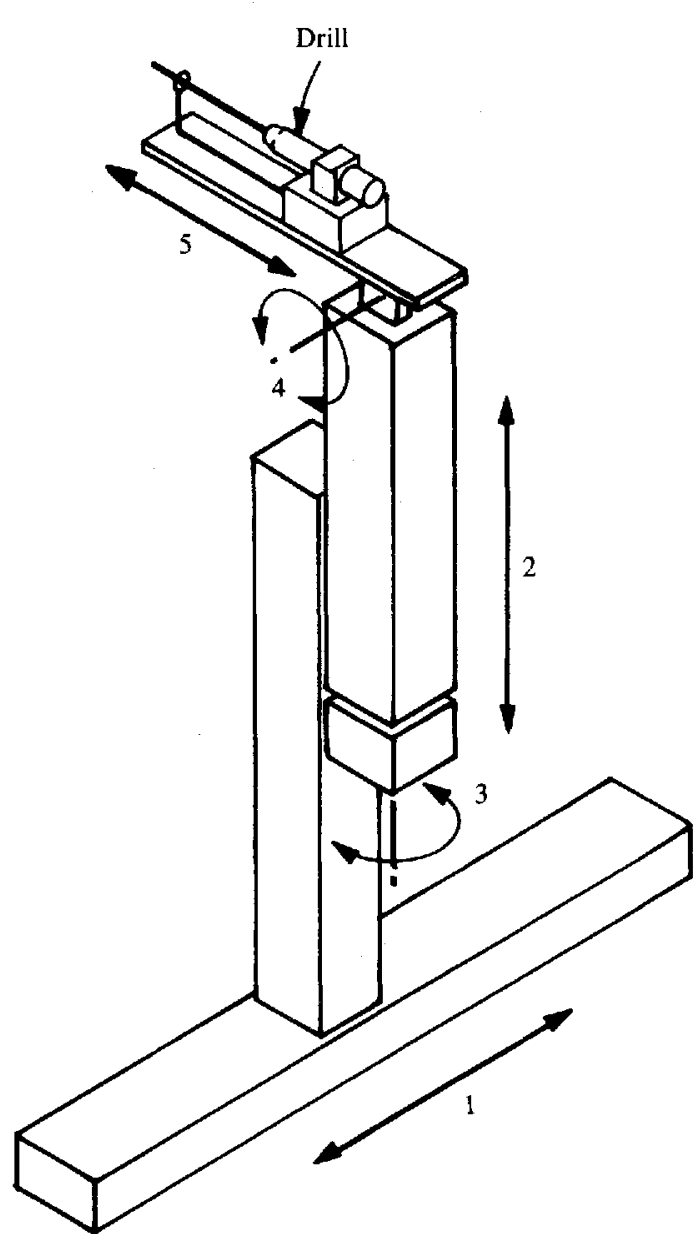

Fig. 5 Schematic diagram of robot

preliminary experimental drilling tests using pig femurs varied between $17 \mathrm{~N}$ and $25 \mathrm{~N}$. A design force of $50 \mathrm{~N}$ has therefore been used. Further drilling tests using the prototype manipulator on human (cadaver) bones are necessary in order to establish the maximum value of the forces involved, as well as to assess the performance of this robotic system. A standard industrial air drill is being used currently for laboratory trials. Further trials using a surgical-type drill will be carried out in the near future.

The drill-holder unit which is mounted on the drill feed actuator has been fitted with a force sensor and a drill bit guide. The force sensor allows the monitoring of the drilling force. This force is also used in conjunction with the drill feed position measurement to form part of the safety protocol as explained in Section 4. The drill bit guide replaces the hand-held guide used in current non-robotic-assisted practices to prevent the drill bit from slipping on the surface of the bone, the drill bit guide travels with the drill bit but stops when it comes in contact with the bone. It is designed to exert only a very small force on the bone while in contact.

\subsection{Vision interface}

The purpose of any three-dimensional robotic vision system is to extract accurate coordinate data from two, or more, digital images. For this unique surgical application, these images are obtained by digitizing the video signal transmitted from a standard mobile C-arm image-intensifier/television system. However, before measurements can be extracted, correction for image distortion (that is, internal calibration) must be applied, followed by external calibration with respect to an arbitrarily defined frame of reference.

The principle of operation of the electron optics of an image-intensifier (I-I) unit requires an almost spherical input phosphor. However, in order to be compatible with television cameras the output phosphor is generally flat. The projection of an X-ray image on to a curved surface followed by a mapping on to a flat output results in a form of spatial distortion commonly referred to as 'pincushion distortion' (8). The fluoroscopic images obtained from an I-I/television system are therefore noticeably distorted, preventing the direct acquisition of quantitative data. A system specific internal calibration process is therefore carried out in order to compensate for non-linearities. In practice, this generally involves the acquisition of calibration images of a rectilinear grid pattern followed by software correction of distortion using either a global polynomial function (9), or a piecewise affine transformation $(\mathbf{1 0}, \mathbf{1 1})$.

External calibration is achieved via the introduction of radio-opaque reference markers, such as ball bearings and lead-wire crosses, into the usual $\mathrm{X}$-ray views. In practice, locating these reference markers, or 'control points', will require the use of a Perspex frame located around the body part of interest. As the actual spacing of the markers is known, it is possible to model the transformation from world to measured image display coordinates.

Once external calibration has taken place, a threedimensional triangulation-based reconstruction of the location of any point of interest may then be performed, providing the image coordinates of this point can be extracted from two calibrated images. Therefore, the vision interface defines the location of the bone with respect to an intermediate frame of reference (Perspex frame), and in doing so allows the required object (bone) to robot transformation to be achieved once the robot has also been calibrated to this intermediate frame as discussed in Section 3.

Due to a limited access to X-ray equipment and the obvious safety risks, the $\mathrm{X}$-ray vision interface has been simulated using CCD cameras for the purpose of initial laboratory trials. Although obvious differences exist between X-ray and visual imaging, the basic concepts, such as the need to overcome image distortion, remain the same. Given that off-the-shelf, non-metric equipment has been used to facilitate this simulation, and existing $\mathrm{C}$-arms and clinical $\mathrm{X}$-ray views are ultimately to be used, modelling of the imaging process by the obvious stereo techniques was not possible. More versatile techniques based upon the direct linear transformation (DLT) and the two-plane calibration method (12) have therefore been investigated. Although these methods are derived from the collinearity condition and as such only partially compensate for lens distortion, moderate accuracy ( $\pm 1.0 \mathrm{~mm}$ absolute error) is easily obtained. When the application calls for sub-millimetre precision, these linear techniques can be used to provide 'initial guess' values for a second non-linear iterative stage (13). The use of sub-pixel registration techniques is also currently under investigation as a means of improving calibration accuracy. 
In order to minimize measurement errors, more control points than are strictly required to form a solution are used during calibration. This degree of redundancy, caused by over sampling, results in an over-determined system of equations. The calibration problem, therefore, calls for a 'best fit' type of solution, which is provided by any number of least-squares techniques. The singular value and $\mathrm{Q}-\mathrm{R}$ decomposition methods have both been successfully applied during this study (14).

In addition to supplying the manipulator controller with accurate drill path data, the vision system also forms an important part of the surgeon's interface. The frame grabber card used to digitize the C-arm output also allows the display of annotated images on the DIPS monitor. The surgeon can therefore be shown the posteroanterior (PA) X-ray image, allowing him or her to indicate the required drill path using a sterilized mouse. The two-dimensional information content of the indicated PA line is then utilized by the imageprocessing software to aid the surgeon's selection of the ideal drill path on the lateral (second) view. In this way, the present requirement for the surgeon to 'think' in three dimensions can be greatly reduced.

As well as allowing the surgeon to input information into the system, the DIPS monitor can be used to display the progress of the drill bit without the need for fluoroscopy. This would be achieved by converting the robot's positional feedback readings into the Perspex frame's system, thus allowing the drill bit location to be displayed.

\section{SAFETY CONSIDERATIONS}

Unlike industrial robots, medical robots operate in contact with, or in very close proximity to, people. The safety requirements are thus different.

It is not advisable, for safety reasons, to use commercial robots as these are prone to making unexpected movements. One wrong movement could have disastrous consequences. Modification of commercial robots, which has been the case in some applications of roboticassisted surgery, would be necessary in order to incorporate the required safety features. Modification of commercial robots is, however, not the optimal approach to robotic-assisted surgery.

The overriding design constraint of medical robotic systems is the safety of the patient, the surgeon and the theatre staff. A mechatronics approach to the design process ensures that safety issues are addressed at the outset in the mechanical design, interface design and software development. Sterility issues have also been taken into account at the outset in the mechanical design.

The surgical procedures for which this robotic device is aimed involve the instrument/tool-holder (or drillholder in this case) moving only in a straight line and to a predetermined extent. One of the safety features of this robotic device is that once the correct orientation of the drill bit (or tool-holder) is obtained, only the linear motion of the drill-holder is permitted, with the rest of the joints locked in position.

A hand-held deadman's handle is provided for the surgeon to ensure that no automatic movement of the manipulator joints is possible unless it is pressed. The

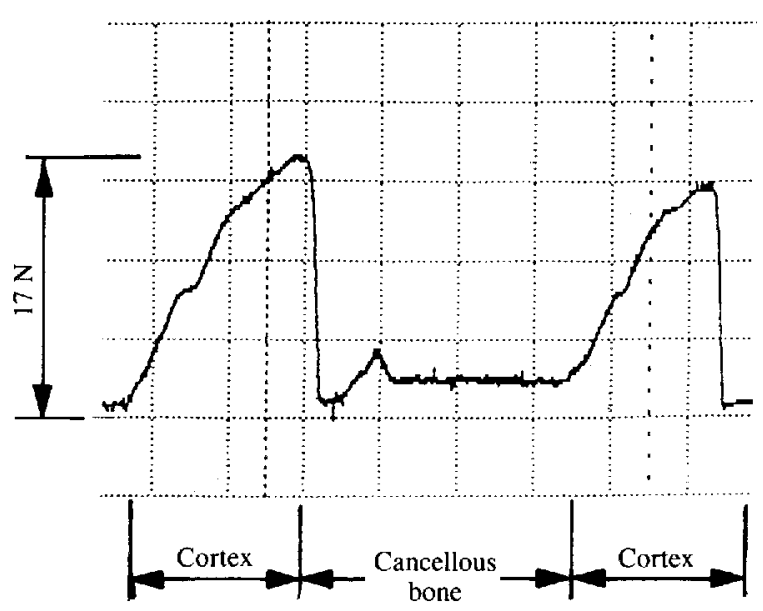

Fig. 6 Drilling force profile at the middle section of a pig femur (feed rate $=2 \mathrm{~mm} / \mathrm{s}$, drilling speed $=3300$ $\mathrm{r} / \mathrm{min}$ )

deadman's handle, which complements the mechanical and electrical safety implements, provides the surgeon with the overall control and the surgeon can safely interrupt the drilling process should it be necessary or when desired to check the drill bit path.

The safety features of the robotic system are extended in this project to prevent damage to the patient caused by the penetration of the drill into the body cavity. In such an accident it is possible that the patient's bladder, blood vessels or bowel could be perforated, often with fatal consequences. This type of accident, though not common, has occurred in existing practices and its complete prevention is another benefit to be gained from the application of robotic assistance.

The drilling depth can be determined, and displayed on the DIPS monitor, as explained in Subsection 3.2. The desired insertion depth, which is established in the calibration procedure, is used to control the drill feed (which is measured using a linear potentiometer). An error in the feed measurement could have a disastrous outcome. A force sensor has been introduced for the measurement of the drill thrust force. Qualitative monitoring of this force helps to determine the 'rough' position of the drill bit tip. Porcine femurs have been used to investigate this procedure.

Figure 6 shows the drilling force profile of the middle section of a pig femur using a surgical guide-wire orthogonal to the long axis of the bone. It can be seen, as expected, that a large force is measured in the cortical bone with a sharp drop at the cortical-cancellous bone interface. The profile of the drill thrust force can, therefore, be incorporated in the safety protocol as an additional safety feature. Numerous drilling tests are, however, still needed to determine (qualitatively) the profile of the drill force at different parts of the femur and for different angles between bone and guide-wire.

\section{DISCUSSION}

The application of computer-controlled robotic technology to surgical procedures dates back to the mid 1980 s. The majority of the earlier systems utilized a modified commercial robot, interfaced with a high resolution three-dimensional imaging device (CT or MRI scanner), to perform precision tool positioning 
tasks. Completion of the invasive stages of the operation was then performed manually by the surgeon using the robot as an instrument guide. Given their non-invasive nature, these robotic surgeon assistants avoided many of the more difficult safety issues. However, extensive modifications to the commercial robots were still required to provide adequate safety fatures. A prime example of these early applications is the stereotactic neurosurgery system implemented by Kwoh et al. (15) which was first used on a human patient in 1985 .

In recent years, as the acceptance of robotic technology within the medical community has grown, a number of invasive applications have been attempted. Acknowledging the shortcomings of general-purpose industrial robots, several researchers have found it necessary to develop purpose-built manipulators in order to satisfy the safety requirements of these more demanding procedures. A new breed of custom-built surgical robots has therefore emerged, such as the Imperial College 'SARP' (surgical assistance robot for prostatectomy) system (16). This robotic system was first used on a human patient in March 1991. A prototype robot 'Minerva' (17) is also currently being developed, at the Swiss Federal Institute of Technology, with the aim of performing entire stereotactic interventions, including skin and bone penetration.

Attempts have also been made to eliminate the expensive dependence upon dedicated CT/MRI imaging for robot 'referencing'. A team at Grenoble University Hospital, France, (18) has performed over 200 successful X-ray guided non-invasive robotic-assisted neurosurgery procedures on human patients using the Perspex reference frame method described in Subsection 3.2.

To date there are no robotic assistant systems for use in the repair of femoral fractures. However, other orthopaedic procedures involving the femur have been successfully investigated. The most widely publicized of these applications is the development, at the University of California, at Davis (UCD), of a computer-controlled robotic system for use in total hip replacement (THR) procedures (19). This system is used to machine a cavity in the proximal femur at the correct position, matching the exact size and shape of a cementless artificial hip prosthesis. Successful clinical trials of this robot, involving human patients, have been in progress since November 1992. A similar robot-aided joint replacement procedure, total knee arthroplasty (TKA), is also under investigation at the University of Washington, Seattle (20).

In order to gain acceptance by the medical community, the current investigation into robotic-assisted fracture fixation has followed the established progression route and as such has been divided into two stages. An initial, feasibility study, stage is being undertaken to prove the principle of robotic-assisted internal fixation of femoral fractures. This involves the design, manufacture and integration of the different parts of a prototype image-guided robotic system. Laboratory trials and a full safety evaluation will also be conducted as part of this preliminary stage. Stage two of the research programme will then be concerned with the clinical evaluation of the system and will include clinical trials followed by further upgrading of the robotic system.
The success of the present investigation is heavily dependent upon practical solutions being obtained to the technical problems imposed by the robot's unique operating theatre working environment. In common with all robotic surgery applications, the critical areas of patient, surgeon and theatre staff safety, sterility and compatibility with existing equipment have had to be addressed. The development of accurate 'referencing' and calibration techniques, for both the robot and the digital X-ray vision system, has also been necessary. The adoption of the multi-disciplinary mechatronics design philosophy has, however, significantly aided the progress of this development work.

Due to limited medical resources, the commercial success of this and other robotic surgery applications will ultimately depend upon the cost of the overall system.

\section{CONCLUSIONS}

The benefits obtained in using a robotic system to assist orthopaedic surgeons in the correct placement of internal fixation devices have been discussed with reference to a Loughborough prototype manipulator designed for laboratory trials. Repair of femoral neck, trochanteric and shaft fractures have been used as exemplar orthopaedic procedures to highlight these benefits.

By the correct deployment of the robotic system it is possible to achieve accurate placement of internal fixation devices, and thus improve the success rate of the orthopaedic surgical repairs, as well as remove the surgical personnel from hazardous proximity to $X$-ray sources. The latter is of great benefit since, unlike the patient, the surgeon suffers repeated $\mathrm{X}$-ray exposure in the course of his or her duties.

\section{ACKNOWLEDGEMENTS}

This research project has been supported by the Wishbone Trust of the British Orthopaedic Association. The authors are grateful for the financial support provided. This work is carried out in collaboration with $\mathrm{Mr} \mathbf{R}$. G. Shedden, Consultant Orthopaedic Surgeon, Mayday University Hospital, Surrey, England.

The authors wish to thank Mr F. R. Ong for his contributions to the experimental tests.

\section{REFERENCES}

1 Sugarman, I. D., Adam, I. and Bunker, T. D. Radiation dosage during AO locking femoral nailing. Injury, 1988, 19, 336-8.

2 Levin, P. E., Schoen, R. W. and Browner, B. D. Radiation exposure to the surgeon during closed intramedullary nailing. $J$. Bone $J t$ Surg., June 1987, 69-A(5), 761-766.

3 Bouazza-Marouf, K. and Hewit, J. R. Robotic repair of fractured femur neck. Proceedings of a seminar on Robotics in keyhole surgery, Institution of Mechanical Engineers, London, 5 May 1993.

4 Preising, B., Hsia, T. C. and Mittelstadt, B. A literature review: robots in medicine. IEEE Engng in Medicine and Biol., June 1991, 13-22.

5 Kassler, M. Robotics for healthcare: a review of the literature. Robotica, November-December 1993, 11, 495-516.

6 Dandy, D. J. Essential orthopaedics and trauma, 1992 (Churchill Livingstone)

7 Kempf, I., Grosse, A. and Beck, G. Closed locked intramedullary nailing-its application to comminuted fractures of the femur. $J$. Bone Jt Surg. June 1985, 67(5), 709-720.

8 Reimann, G. A. and Flynn, M. J. Automatic distortion correction of X-ray image intensifier images. Conference Record 1992 IEEE 
Nuclear Science Symposium and Medical Imaging Conference, Vol. 2, 1992, Ch. 438, pp. 1339-1341.

9 Rudin, S., Bednarek, D. R. and Wong, R. Accurate characterisation of image-intensifier distortion. Med. Physics., NovemberDecember 1991, 18(6), 1145-1151.

10 Boone, J. M., Seibert, J. A., Barrett, W. A. and Blood, E. A. Analysis and correction of imperfections in the image intensifierTV-digitizer imaging chain. Med. Physics, March-April 1991, 18(2), 236-242.

11 Butler, D. A. and Pierson, P. K. A distortion-correction scheme for industrial machine-vision applications. IEEE Trans. on Robotics and Automation, August 1991, 7(4), 546-51.

12 Martins, H. A., Birk, J. R. and Kelley, R. B. Camera models based on data from two calibration planes. Computer Graphics and Image Processing, 1981, 17, 173-80.

13 Tsai, R. Y. An efficient and accurate camera calibration technique for three-dimensional machine vision. Proceedings of the IEEE International Conference on Computer vision and pattern recognition, 1986, 364-374.

14 Nash, J. C. Compact numerical methods for computers: linear algebra and function minimisation, 1980 (Adam Hilger Limited).

15 Kwoh, Y. S., Hou, J., Jonckheere, E. A. and Hayati, S. A robot with improved absolute positioning accuracy for CT guided stereotactic brain surgery. IEEE Trans. on Biomed. Engng, February $1988,35,153-160$.

$16 \mathrm{Ng}$, W. S., Davies, B. L., Hibberd, R. D. and Timoney, A. G. Robotic surgery: a first-hand experience in transurethral resection of the prostate. IEEE Engng in Medicine \& Biol. Magazine, 1993, 12(1), 120-125.

17 Glauser, D., Flury, P. and Burckhardt, C. W. Mechanical concept of the neurosurgical robot 'Minerva'. Robotica, NovemberDecember 1993, 11, 495-516.

18 Lavallee, S., Cinquin, P., Demongeot, J., Benabid, A. L., Marque, I. and Djaid, M. Computer assisted interventionist imaging: the instance of stereotactic brain surgery. Proceedings of the Sixth Conference on Medical informatics, MEDINFO ' 89 , Beijing, China, 16-20 October 1989, 613-617.

19 Taylor, R. H., et al. An image-directed robotic system for precise orthopaedic surgery. Proceedings of the Twelfth Annual International Conference of the IEEE Enyineering in Medicine and Biology Society, 1990, 12(5), 1928-1930.

20 Matsen, F. A., Garbini, J. L., Sidles, J. A., Pratt, B., Baumgarten, T. K. and Kiaura, R. Robotic assistance in orthopaedics: a proof of principle using distal femoral arthroplasty. Clin. Orthop. Related Res., 1993, Number 296, 178-186. 